\title{
Performance of the DAMPE silicon-tungsten tracker-converter during the first 5 years of in-orbit operations
}

\section{Perrina, ${ }^{a, 1, *}$ P. Azzarello, ${ }^{a}$ E. Catanzani, ${ }^{b, c}$ A. Tykhonov ${ }^{a}$ and $\mathbf{X}$. Wu $\mathbf{W u}^{a}$ on behalf of the DAMPE Collaboration}

(a complete list of authors can be found at the end of the proceedings)

${ }^{a}$ Département de Physique Nucléaire et Corpusculaire (DPNC), Université de Genève, CH-1211 Genève 4, Switzerland

${ }^{b}$ Department of Physics and Geology, University of Perugia, I-06123 Perugia, Italy

${ }^{c}$ INFN Sezione di Perugia, via A. Pascoli, I-06123 Perugia, Italy

E-mail: Chiara.Perrina@epfl.ch

Since its launch, in December 2015, the satellite-based DAMPE (DArk Matter Particle Explorer) particle detector is taking data smoothly. The Silicon-Tungsten tracKer-converter (STK) of DAMPE consists of six tracking planes $(6 \mathrm{x}, 6 \mathrm{y})$ of single-sided silicon micro-strip detectors mounted on seven support trays. The STK is able to measure the charge and precisely reconstruct the track of traversing charged particles. Tungsten plates ( $1 \mathrm{~mm}$ thick) are integrated in the second, third and fourth tray from the top to serve as $\gamma \rightarrow e^{+} e^{-}$converters. Commissioned rapidly after the launch, the STK is running extremely well since then. The STK in-orbit calibration and performance during its first more than 5 years of operation, including the noise behaviour and the thermal and mechanical stability, are presented in this contribution.

$37^{\text {th }}$ International Cosmic Ray Conference (ICRC 2021)

July 12 th - 23rd, 2021

Online - Berlin, Germany

\footnotetext{
${ }^{1}$ Also at Institute of Physics, Ecole Polytechnique Fédérale de Lausanne (EPFL), CH-1015 Lausanne, Switzerland

${ }^{*}$ Presenter
} 


\section{Introduction}

The DArk Matter Particle Explorer (DAMPE) is a satellite-based experiment for the detection of charged cosmic rays and gamma rays. It was launched on December 17, 2015 from the Jiuquan Satellite Launch Center in the Gobi Desert. DAMPE operates in a Sun-synchronous orbit around Earth at an altitude of about $500 \mathrm{~km}$, with a period of about 95 minutes and an inclination of around $97^{\circ}$. Thanks to its four subdetectors, DAMPE detects electrons, positrons and photons from few $\mathrm{GeV}$ to $10 \mathrm{TeV}$, as well as protons and heavier nuclei from $10 \mathrm{GeV}$ to $100 \mathrm{TeV}$, with excellent energy and angular resolutions [1]. The DAMPE subdetectors are (Fig. 1):

1. the Plastic Scintillator Detector (PSD), which measures the absolute value of the charge $(|Z|)$ of cosmic rays and acts as an anti-coincidence system for charged particles in the gamma-ray detection;

2. the Silicon-Tungsten tracKer-converter (STK), which is described in detail in the next section;

3. the imaging calorimeter made of 14 layers of Bismuth Germanium Oxide (BGO) crystals in a hodoscopic arrangement with a total thickness of about 32 radiation lengths, which measures the energy of the incoming particles and can distinguish electrons from hadrons;

4. the NeUtron Detector (NUD), a boron-doped plastic scintillator detecting delayed neutrons coming from the hadronic interactions at high energies, which boosts the electron/hadron separation power.

DAMPE is taking data smoothly since its launch, except for two days in May 2021, recording about five million events per day.

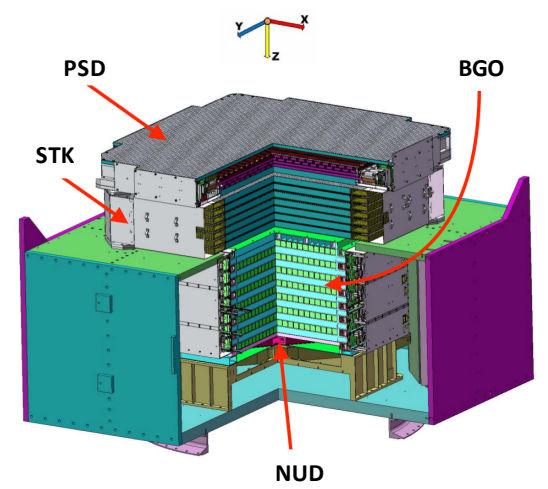

Figure 1: Schematic view of the DAMPE detector. From the top, the 4 subdetectors are: the plastic scintillator detector (PSD), the silicon-tungsten tracker-converter (STK), the bismuth germanate calorimeter (BGO) and the neutron detector (NUD).

\section{The Silicon-Tungsten tracKer-converter (STK)}

The DAMPE Silicon-Tungsten tracKer-converter (STK) [2] was designed for the purpose of reconstructing the trajectories and the charge absolute value $(|Z|)$ of charged cosmic rays, 
favour the conversion of gamma-rays and reconstruct the tracks of the generated electrons and positrons. The STK was rapidly commissioned after the launch and the data acquisition started on December 30, 2015, less than two weeks after the launch. The STK has been functioning extremely well since then, giving proof of its essential role in the physics results achieved by the DAMPE Collaboration [3-5].

The DAMPE STK (Fig. 2) consists of 6 tracking planes, for 6 independent measurements of the position ( $\mathrm{x}, \mathrm{y})$ of a charged particle traversing the STK. Each tracking plane consists of two layers of single-sided Silicon micro-Strip Detectors (SSDs) measuring the two orthogonal views perpendicular to the pointing direction of the detector. A layer is a matrix of $8 \times 2$ ladders, each ladder (Fig. 3) consists of 4 single-sided AC-coupled SSDs, daisy-chained via micro-wire bonds. The SSDs, produced by Hamamatsu Photonics, have a thickness of $320 \mu \mathrm{m}$ and a sensitive area of $9.29 \mathrm{~cm} \times 9.29 \mathrm{~cm}$ segmented into 768 strips with a pitch of $121 \mu \mathrm{m}$. To limit power consumption, electronics density and transferred data, only one every second strip is read out, therefore the STK has 73728 readout channels. The 384 channels of each ladder are read out by 6 VA140 ASIC chips made by IDEAS. The 192 ladders are read out in groups of 24 by 8 data acquisition Tracker Readout Boards (TRBs) located on the sides. The tracking layers are mounted on 7 support trays, the top and the bottom trays are equipped only on the side facing the five internal trays which are equipped on both sides. A support tray consists of a core of aluminium honeycomb sandwiched between two sheets of Carbon Fibre Reinforced Polymer (CFRP). To favour the photon conversion into electron-positron pairs, in the second, third and fourth tray, $1 \mathrm{~mm}$ thick tungsten plates are glued on the lower CFRP sheet, giving a total thickness equivalent to about one radiation length. The total power consumption of the STK is about $90 \mathrm{~W}$.
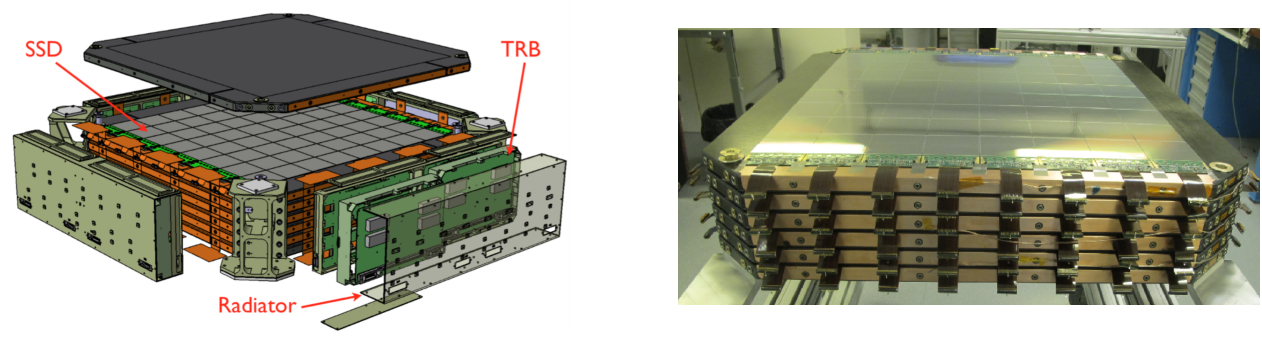

Figure 2: Left: Exploded view of the STK. Each tracking layer is made of 64 silicon micro-strip detectors (SSDs). On each side of the STK there are two data acquisition boards (TRBs) and a radiator which dissipates the heat produced by the electronics. Right: Photograph of the STK before the assembly of the last support tray.

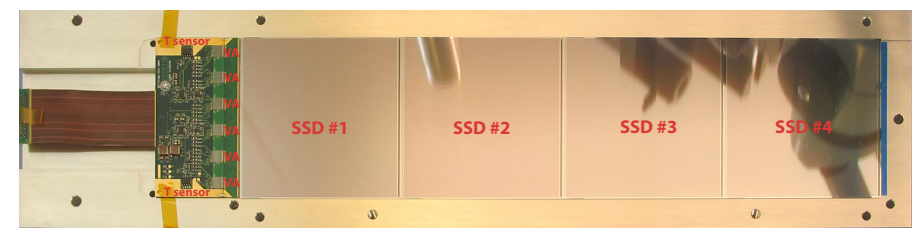

Figure 3: Photograph of one ladder. The 4 SSDs are daisy-chained via micro-wire bonds. The front-end electronics hosts 6 VA140 ASICs for the readout of 384 channels and 2 temperature probes. 


\section{The STK in-orbit performance}

\subsection{Noise and temperature behaviour}

The STK has always revealed an excellent noise stability. The left panel of Fig. 4 shows the distribution of the channel noise at the beginning of the mission (January 1, 2016) and recently (June 15, 2021). The two distributions have a mean $<3$ ADC, coincident with their most probable value, and they are very similar, denoting the excellent STK noise stability. Since the signal for a Minimum Ionizing Particle (MIP) hitting a readout strip is $\sim 52$ ADC, the achieved signal/noise ratio is $\sim 18$. Another indicator of the excellent quality of the STK is the fraction of good channels, defined conservatively as the channels with a noise $\sigma \leqslant 5$ ADC. The right panel of Fig. 4 shows the time evolution of the good channel fraction together with the up to date time evolution of the ratio of channels with a noise $\sigma \in(5,10] \mathrm{ADC}$ and $\sigma>10 \mathrm{ADC}$. At the beginning of the data acquisition, the $99.55 \%$ of the channels was good, this fraction increased over time thanks to the stabilization in space and since two years its value is stable around $99.74 \%$.
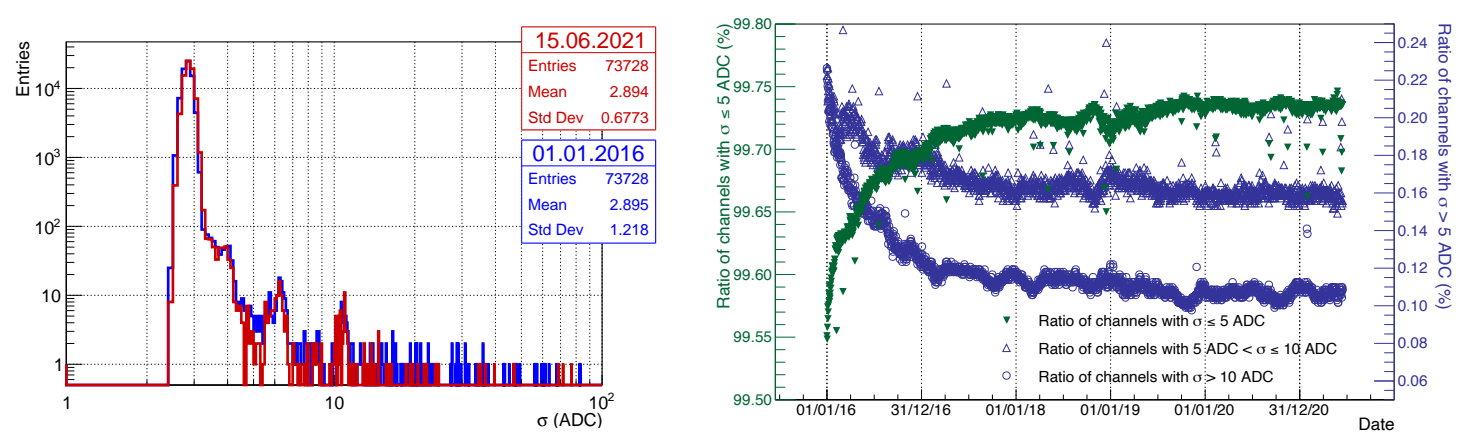

Figure 4: Right: Noise distribution of the 73728 STK readout channels at the beginning of the mission (January 1, 2016) and recently (June 15, 2021). Left: Time evolution of the ratio of channels with noise $\sigma \leqslant 5$ ADC (green full triangles), $\sigma \in(5,10]$ ADC (blue empty triangles) and $\sigma>10$ ADC (blue empty circles).

The excellent STK noise stability is correlated to the high STK temperature stability. The latter is due to the robustness of the mechanical design and the Sun-synchronous orbit. Fig. 5 shows the time evolution of the STK mean noise and the STK mean temperature. The STK mean noise is computed as the mean of all the channel noises, while the STK mean temperature is the mean of all the temperatures measured by two probes placed on the front-end electronics of each ladder. The STK mean temperature is very stable, with a daily variation much lower than $0.1^{\circ} \mathrm{C}$. The evident periodicity is due to the seasonal variation of the satellite orbit, moderated by the Earth's shadow from May to July. As a result the STK mean noise is very stable with a maximum variation of 0.04 ADC. For two days, from May 23 to 24, 2021 the payload was not powered. In the right panel of Fig. 5 we can see that only six days after the detector was powered on again, both the noise and the temperature were back at the expected levels. The correlation between the noise and the temperature can be quantified performing the linear fits shown in Fig. 6. The STK mean noise is increasing slightly more than the STK mean temperature, from $0.008 \mathrm{ADC} /{ }^{\circ} \mathrm{C}$ to $0.01 \mathrm{ADC} /{ }^{\circ} \mathrm{C}$ in five years, possibly because of a still negligible radiation damage of the silicon detectors. 


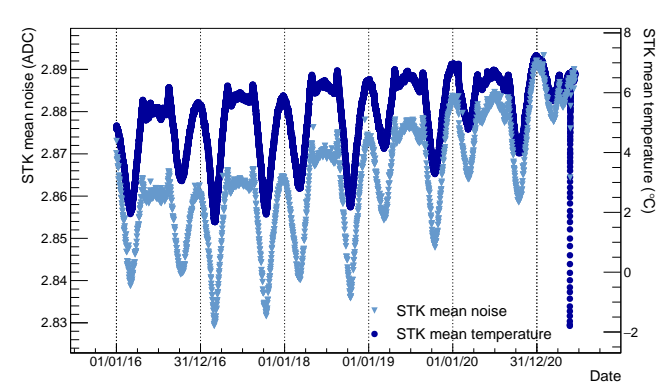

(a) From the beginning of the data taking.

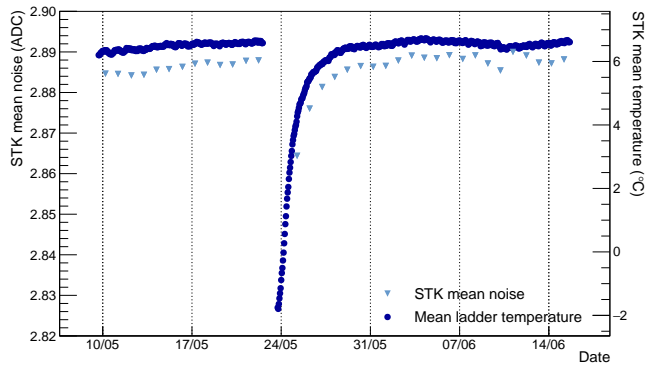

(b) From May 9, 2021.

Figure 5: Up to date time evolution of the STK mean noise (light blue triangles) and of the STK mean temperature (dark blue dots).
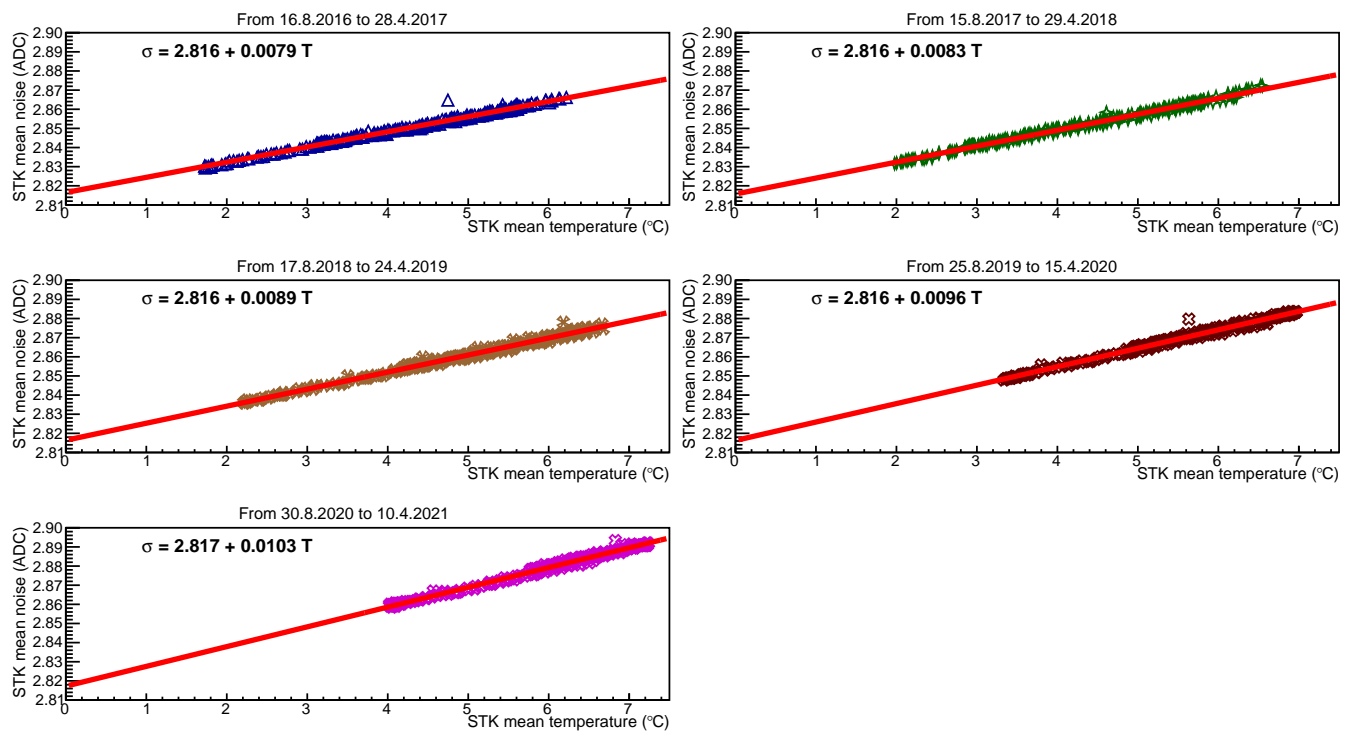

Figure 6: STK mean noise as a function of the STK mean temperature from August to April for every year, excluding the period when the satellite is in the Earth's shadow.

\subsection{Alignment and position resolution}

The position resolution of the silicon sensors, achieved with the analog readout that takes into account the charge sharing on the non-readout strips, is less than $50 \mu \mathrm{m}$ for incidence angles lower than $40^{\circ}$, and much lower than the $70 \mu \mathrm{m}$ achievable with a digital position finding algorithm below $70^{\circ}$ [2]. The good temperature stability also ensures a good mechanical stability of the STK. The mechanical assembly of the STK has a construction precision of about $100 \mu \mathrm{m}$ that is larger than the position resolution of the silicon sensors, therefore an alignment procedure is needed to correct for the displacement and rotation of each sensor with respect to its nominal position, allowing the full tracker potential to be exploited [6]. Five alignment parameters are considered for each silicon sensor: $\Delta_{x} / \Delta_{y}, \Delta_{z}, \theta_{x}, \theta_{y}, \theta_{z}$. They correspond to the two offsets and three rotation angles of the silicon sensor, for a total of 3840 alignment parameters. As an example, Fig. 7 shows the time evolution of the variation $(\delta \mathrm{z})$ of the average $\mathrm{z}$ position of the sensors of each STK tracking layer with respect to the average $z$ position of the sensors of the first layer $(1 \mathrm{x}$, tray 1$)$. The change of 
this variation with time can be explained by two main effects: humidity release and temperature variation. We observe in the beginning of in-orbit operations the contraction of the support trays, due to the humidity release process, then a correlation with the temperature. The importance of the

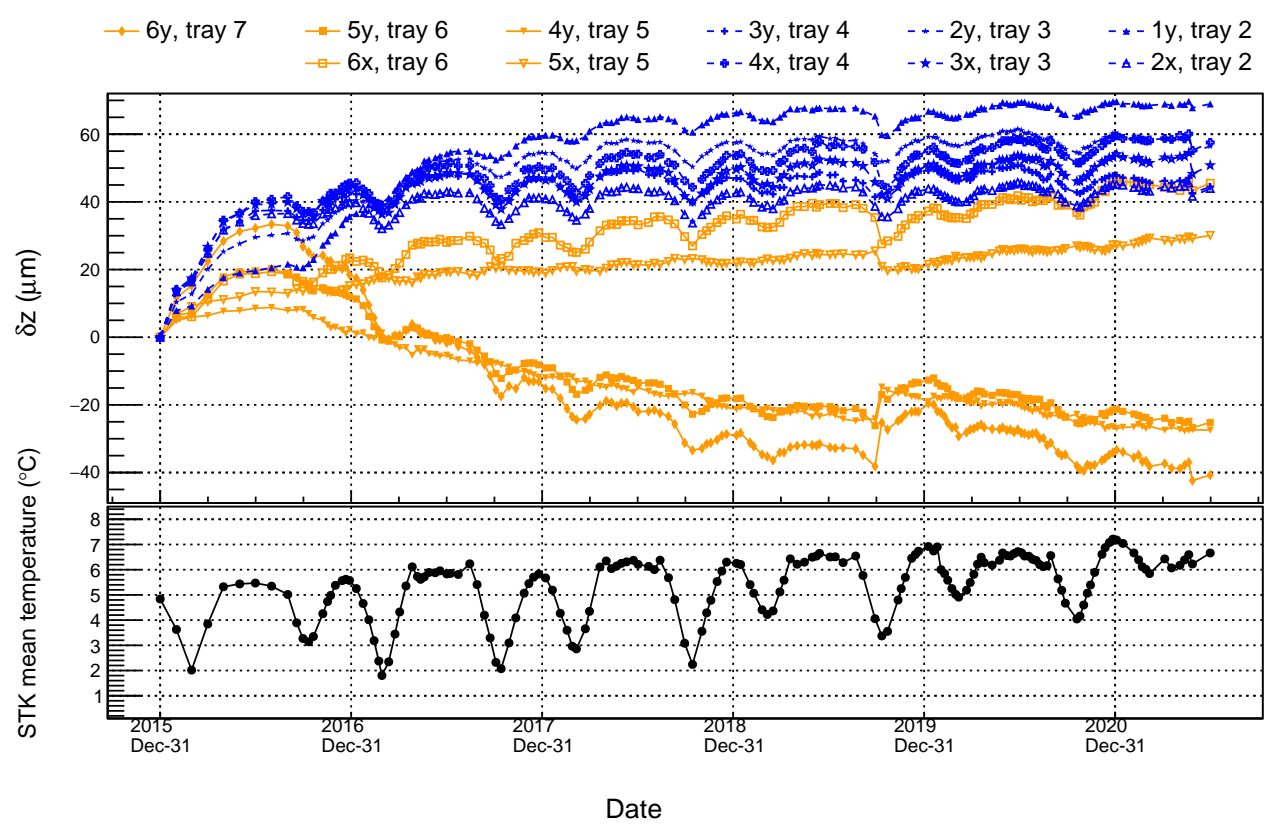

Figure 7: The variation of the average z position of each STK tracking layer with respect to the first layer ( $1 \mathrm{x}$, tray 1$)$ as a function of time (top), and the average temperature of the STK, measured by the temperature probes of all the ladders (bottom). A realignment is done once every two weeks. The dashed/blue curves correspond to the trays hosting tungsten plates.

alignment, performed once every two weeks, is demonstrated in Fig. 8, where the optimal position resolution of the 6 STK $x$ layers, as a function of the time is shown for different incidence angles of the reconstructed tracks. After the alignment, the optimal position resolution for incidence angles below $50^{\circ}$ is much lower than $70 \mu \mathrm{m}$. Fig. 9 shows the variation of the optimal position resolution as a function of time. Thanks to the bi-weekly updates of the alignment parameters, the optimal position resolution remains stable and below $6 \%$ for all STK layers and particle incidence angles.

\section{Conclusions}

The Silicon-Tungsten tracKer-converter (STK) of the DAMPE mission, based on the robust technology of single-sided silicon strip detectors with analog readout, plays a crucial role in the reconstruction of charged particle tracks, in the gamma-ray detection, and in the cosmic ray charge measurement. The STK is showing an excellent noise, thermal and mechanical stability since the beginning of the data taking. About the totality of channels (99.74\%) are superbly performing, with a noise less than 5 ADC. In-orbit calibration and alignment procedures allow to achieve the best possible position and angular resolutions and long term stability. 

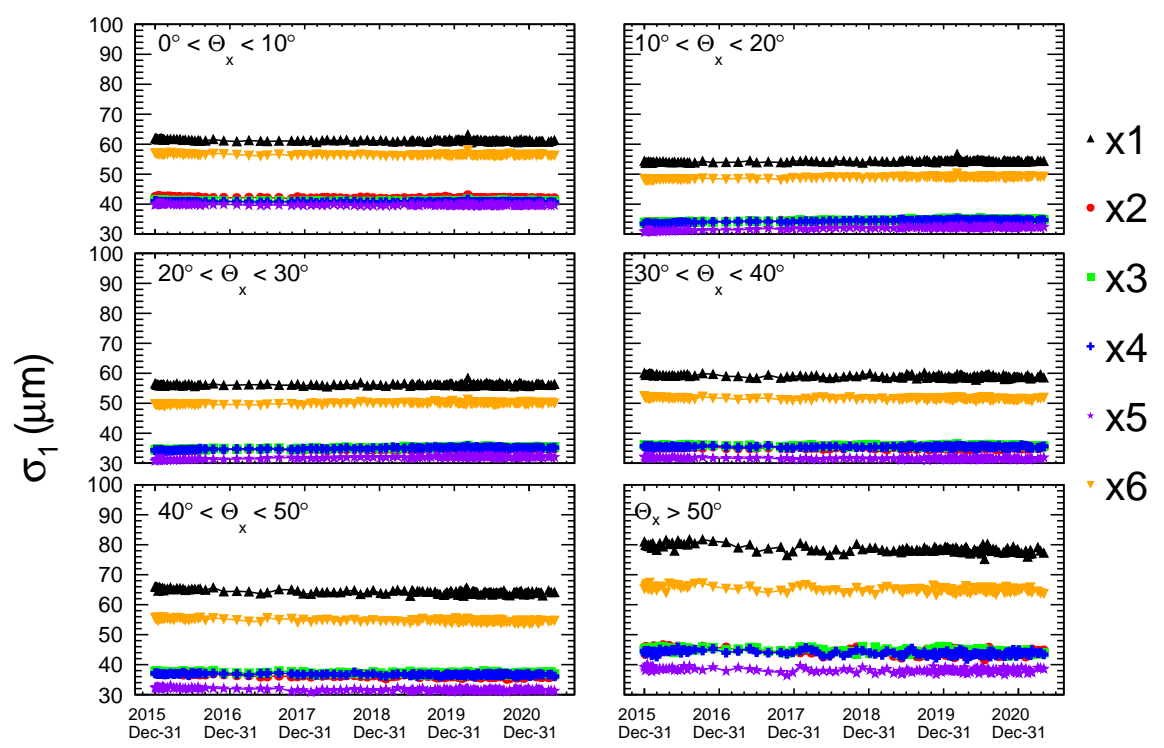

Figure 8: Optimal position resolution for the six $x$ layers at different track inclinations as a function of time. The values are shown for $\mathrm{x}$ layers only, while y layers show a similar behaviour.

\section{Acknowledgments}

The DAMPE mission is funded by the strategic priority science and technology projects in space science of Chinese Academy of Sciences. In China the data analysis is supported by the National Key Research and Development Program of China (No. 2016YFA0400200), the National Natural Science Foundation of China (Nos. 11525313, 11622327, 11722328, 11851305, 11921003 , 12003076, U1738205, U1738206, U1738207, U1738208, U1738127), the strategic priority science and technology projects of Chinese Academy of Sciences (No. XDA15051100), the Young Elite Scientists Sponsorship Program by CAST (No. YESS20160196), and the Program for Innovative Talents and Entrepreneur in Jiangsu. In Europe the activities and data analysis are supported by the Swiss National Science Foundation (SNSF), Switzerland, and the National Institute for Nuclear Physics (INFN), Italy.

\section{References}

[1] J. Chang, G. Ambrosi, Q. An, R. Asfandiyarov, P. Azzarello, P. Bernardini et al., The DArk Matter Particle Explorer mission, Astroparticle Physics 95 (2017) 6.

[2] P. Azzarello et al., The DAMPE silicon-tungsten tracker, Nucl. Instrum. Meth. A 831 (2016) 378.

[3] DAMPE collaboration, Direct detection of a break in the teraelectronvolt cosmic-ray spectrum of electrons and positrons, Nature 552 (2017) 63 [1711. 10981].

[4] DAMPE collaboration, Measurement of the cosmic-ray proton spectrum from $40 \mathrm{GeV}$ to 100 TeV with the DAMPE satellite, Sci. Adv. 5 (2019) eaax3793 [1909 . 12860]. 

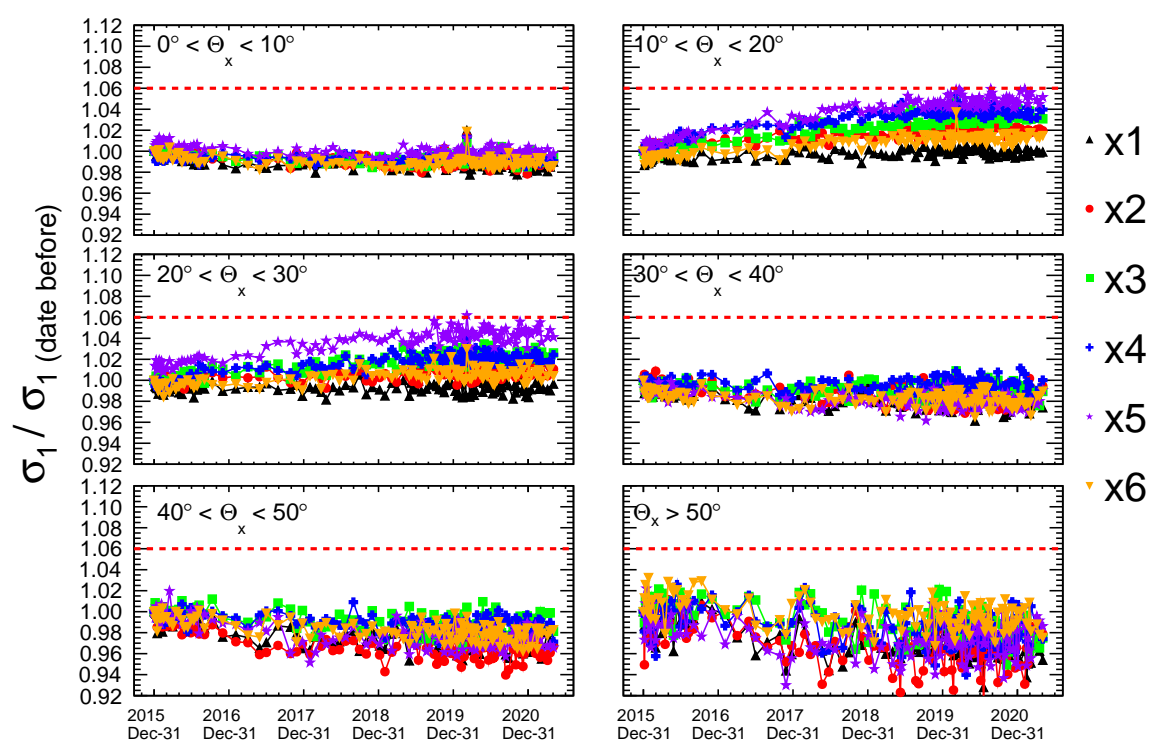

Figure 9: The variation of the optimal position resolution for the six $x$ layers at different track inclinations as a function of time. The values are shown for $\mathrm{x}$ layers only, while y layers show similar behaviour. Horizontal dashed lines are shown to indicate the $6 \%$ deviation from the initial values. The alignment is performed once every two weeks.

[5] DAMPE collaboration, Measurement of the cosmic ray helium energy spectrum from $70 \mathrm{GeV}$ to $80 \mathrm{TeV}$ with the DAMPE space mission, Phys. Rev. Lett. 126 (2021) 201102.

[6] A. Tykhonov et al., Internal alignment and position resolution of the silicon tracker of DAMPE determined with orbit data, Nuclear Instruments and Methods in Physics Research Section A: Accelerators, Spectrometers, Detectors and Associated Equipment 893 (2018) 43. 


\section{Full Authors List: DAMPE Collaboration}

F. Alemanno ${ }^{1,2}$, Q. An ${ }^{3,4}$, P. Azzarello ${ }^{5}$, F. C. T. Barbato ${ }^{1,2}$, P. Bernardini ${ }^{6,7}$, X. J. Bi ${ }^{8,9}$, M. S. Cai ${ }^{10,11}$, E. Casilli6, ${ }^{6,7}$, E. Catanzani ${ }^{12}$, J. Chang ${ }^{10,11}$, D. Y. Chen ${ }^{10}$, J. L. Chen ${ }^{13}$, Z. F. Chen ${ }^{10,11}$, M. Y. Cui ${ }^{10}$, T. S. Cui ${ }^{14}$, Y. X. Cui ${ }^{10,11}$, H. T. Dai ${ }^{3,4}$, A. De Benedittis ${ }^{6,7}$, I. De Mitri ${ }^{1,2}$, F. de Palma ${ }^{6,7}$, M. Deliyergiyev ${ }^{5}$, M. Di Santo ${ }^{1,2}$, Q. Ding ${ }^{10,11}$, T. K. Dong ${ }^{10}$, Z. X. Dong ${ }^{14}$, G. Donvito ${ }^{15}$, D. Droz ${ }^{5}$, J. L. Duan ${ }^{13}$, K. K. Duan ${ }^{10}$, D. D’Urso ${ }^{12,1}$, R. R. Fan ${ }^{8}$, Y. Z. Fan ${ }^{10,11}$, K. Fang ${ }^{8}$, F. Fang ${ }^{13}$, C. Q. Feng ${ }^{3,4}$, L. Feng ${ }^{10}$, P. Fusco ${ }^{15,16}$,

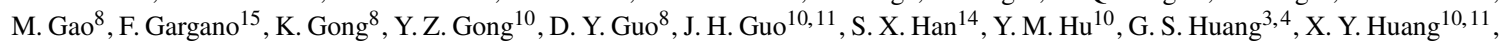
Y. Y. Huang ${ }^{10}$, M. Ionica ${ }^{12}$, W. Jiang ${ }^{10}$, J. Kong ${ }^{13}$, A. Kotenko ${ }^{5}$, D. Kyratzis ${ }^{1,2}$, S. J. Lei ${ }^{10}$, W. H. Li ${ }^{10,11}$, W. L. Li ${ }^{14}$, X. Li $^{10}$, X. Q. Li ${ }^{14}$,

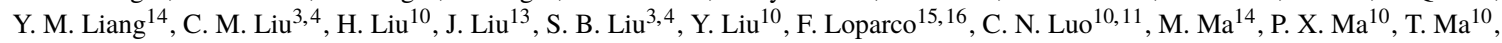
X. Y. $\mathrm{Ma}^{14}$, G. Marsella ${ }^{6,7}{ }_{2}$, M. N. Mazziotta ${ }^{15}$, D. $\mathrm{Mo}^{13}$, X. Y. Niu ${ }^{13}$, X. Pan $^{10,11}$, A. Parenti ${ }^{1,2}$, W. X. Peng ${ }^{8}$, X. Y. Peng ${ }^{10}$, C. Perrina ${ }^{5,3}$, R. Qiao ${ }^{8}$, J. N. Rao ${ }^{14}$, A. Ruina ${ }^{5}$, M. M. Salinas ${ }^{5}$, G. Z. Shang ${ }^{14}$, W. H. Shen ${ }^{14}$, Z. Q. Shen ${ }^{10}$, Z. T. Shen ${ }^{3,4}$, L. Silveri $^{1,2}$, J. X. Song ${ }^{14}$, M. Stolpovskiy ${ }^{5}$, H. Su ${ }^{13}$, M. Su ${ }^{17}$, H. R. Sun ${ }^{3,4}$, Z. Y. Sun ${ }^{13}$, A. Surdo ${ }^{7}$, X. J. Teng ${ }^{14}$, A. Tykhonov ${ }^{5}$, H. Wang ${ }^{14}$, J. Z. Wang ${ }^{8}$, L. G. Wang ${ }^{14}$, S. Wang ${ }^{10}$, S. X. Wang ${ }^{10}$, X. L. Wang ${ }^{3,4}$, Y. Wang ${ }^{3,4}$, Y. F. Wang ${ }^{3,4}$, Y. Z. Wang ${ }^{10}$, D. M. Wei ${ }^{10,11}$, J. J. Wei ${ }^{10}$, Y. F. Wei ${ }^{3,4}$, D. Wu ${ }^{8}$, J. Wu ${ }^{10,11}$, L. B. Wu ${ }^{1,2}$, S. S. $\mathrm{Wu}^{14}$, X. Wu ${ }^{5}$, Z. Q. Xia ${ }^{10}$, H. T. Xu ${ }^{14}$, Z. H. Xu ${ }^{10,11}$, Z. L. Xu ${ }^{10}$, E. H. Xu ${ }^{3,4}$, Z. Z. Xu ${ }^{3,4}$, G. F. Xue ${ }^{14}$, H. B. Yang ${ }^{13}$, P. Yang ${ }^{13}$, Y. Q. Yang ${ }^{13}$, H. J. Yao ${ }^{13}$, Y. H. Yu ${ }^{13}$, G. W. Yuan ${ }^{10,11}$, Q. Yuan ${ }^{10,11}$, C. Yue ${ }^{10}$, J. J. Zang ${ }^{104}$ S. X. Zhang ${ }^{13}$, W. Z. Zhang ${ }^{14}$, Yan Zhang ${ }^{10}$, Yi Zhang ${ }^{10,11}$, Y. J. Zhang ${ }^{13}$, Y. L. Zhang ${ }^{3,4}$, Y. P. Zhang ${ }^{13}$, Y. Q. Zhang ${ }^{10}$, Z. Zhang ${ }^{10}$, Z. Y. Zhang ${ }^{3,4}$, C. Zhao ${ }^{3,4}$, H. Y. Zhao ${ }^{13}$, X. F. Zhao ${ }^{14}$, C. Y. Zhou ${ }^{14}$, and Y. Zhu ${ }^{14}$

${ }^{1}$ Gran Sasso Science Institute (GSSI), Via Iacobucci 2, I-67100 L'Aquila, Italy

${ }^{2}$ Istituto Nazionale di Fisica Nucleare (INFN) - Laboratori Nazionali del Gran Sasso, I-67100 Assergi, L'Aquila, Italy

${ }^{3}$ State Key Laboratory of Particle Detection and Electronics, University of Science and Technology of China, Hefei 230026, China

${ }^{4}$ Department of Modern Physics, University of Science and Technology of China, Hefei 230026, China

${ }^{5}$ Department of Nuclear and Particle Physics, University of Geneva, CH-1211 Geneva 4, Switzerland

${ }^{6}$ Dipartimento di Matematica e Fisica E. De Giorgi, Università del Salento, I-73100 Lecce, Italy

${ }^{7}$ Istituto Nazionale di Fisica Nucleare (INFN) - Sezione di Lecce, I-73100 Lecce, Italy

${ }^{8}$ Institute of High Energy Physics, Chinese Academy of Sciences, Yuquan Road 19B, Beijing 100049, China

${ }^{9}$ University of Chinese Academy of Sciences, Yuquan Road 19A, Beijing 100049, China

${ }^{10}$ Key Laboratory of Dark Matter and Space Astronomy, Purple Mountain Observatory, Chinese Academy of Sciences, Nanjing 210023 , China

${ }^{11}$ School of Astronomy and Space Science, University of Science and Technology of China, Hefei 230026, China

${ }^{12}$ Istituto Nazionale di Fisica Nucleare (INFN) - Sezione di Perugia, I-06123 Perugia, Italy

${ }^{13}$ Institute of Modern Physics, Chinese Academy of Sciences, Nanchang Road 509, Lanzhou 730000, China

${ }^{14}$ National Space Science Center, Chinese Academy of Sciences, Nanertiao 1, Zhongguancun, Haidian district, Beijing 100190, China

${ }^{15}$ Istituto Nazionale di Fisica Nucleare (INFN) - Sezione di Bari, I-70125 Bari, Italy

${ }^{16}$ Dipartimento di Fisica “M. Merlin” dell’Università e del Politecnico di Bari, I-70126 Bari, Italy

${ }^{17}$ Department of Physics and Laboratory for Space Research, the University of Hong Kong, Pok Fu Lam, Hong Kong SAR, China

${ }^{1}$ Now at Dipartimento di Chimica e Farmacia, Università di Sassari, I-07100 Sassari, Italy.

${ }^{2}$ Now at Dipartimento di Fisica e Chimica "E. Segrè", Università degli Studi di Palermo, via delle Scienze ed. 17, I-90128 Palermo, Italy.

${ }^{3}$ Also at Institute of Physics, Ecole Polytechnique Fédérale de Lausanne (EPFL), CH-1015 Lausanne, Switzerland.

${ }^{4}$ Also at School of Physics and Electronic Engineering, Linyi University, Linyi 276000, China. 